SCIENTIFIC LETTER

\title{
Subclinical pulmonary vein narrowing after ablation for atrial fibrillation
}

\author{
T H Hauser, S B Yeon, S McClennen, G Katsimaglis, K V Kissinger, M E Josephson, N M Rofsky, \\ W J Manning
}

Heart 2005;91:672-673. doi: 10.1136/hrt.2004.039347

A trial fibrillation $(\mathrm{AF})$ is the most common sustained cardiac arrhythmia. The recognition that paroxysmal AF may be initiated by abnormalities in the pulmonary veins (PVs) led to the development of catheter based radiofrequency ablation procedures to isolate the $\mathrm{PV}$ and prevent the recurrence of AF. ${ }^{1}$ Clinical PV stenosis is recognised as an uncommon but severe complication. ${ }^{2}$ Contrast enhanced three dimensional magnetic resonance angiography (CE-MRA) ${ }^{3}$ readily visualises the $\mathrm{PV}$ and may detect changes in PV size before the development of clinical stenosis. ${ }^{4}$ We measured in detail the PV diameter, perimeter, and cross sectional area (CSA) in a consecutive series of patients who underwent CE-MRA before and one month after AF ablation.

\section{METHODS}

The study cohort consisted of 23 consecutive patients with paroxysmal AF who underwent CE-MRA before and after AF ablation from October 2001 to May 2003. The study was approved by the hospital committee on clinical investigation.

AF ablation was performed by a standard method ${ }^{5}$ with mapping of the left atrium and PV by the CARTO system (Biosense Webster Inc, Diamond Bar, California, USA). The PV maximum diameter, perimeter, and CSA were measured before and after the procedure by an observer blinded to the clinical treatment. ${ }^{3}$ The left atrial dimension was measured in the axial plane.

Continuous variables are presented as the mean (SD) and categorical variables are presented as count (percentage). Differences between individual PVs were compared by analysis of variance with the Student-Newman-Keuls adjustment. Serial left atrial and PV measurements were compared by a paired Student's $t$ test. A two sided $p<0.05$ was used to determine significance. Data were analysed with SAS for Windows (version 8.2; SAS Institute, Cary, North Carolina, USA).

The relation between the intensity of radiofrequency ablation and PV size changes was assessed by standard correlation and by comparing those PVs that received more than the median number of radiofrequency ablations with those that received fewer than or equal to the median. The duration of radiofrequency application was analysed in the same way.

\section{RESULTS}

The mean age of the cohort was 54 (11) years and the majority were men $(20(87 \%))$. The mean duration of symptomatic AF was 3.5 (3.8) years. Hypertension was present in $10(43 \%)$, obstructive sleep apnoea in seven (30\%), greater than mild mitral regurgitation in 4 (17\%), and cardiomyopathy in two (7\%). No co-morbidity was present in seven $(30 \%)$. There were no acute complications from the procedure.

A total of 86 PVs were evaluated. PV CE-MRA was performed a median of 32 (interquartile range 29-36) days after ablation. CE-MRA was performed during sinus rhythm in all but two patients, who had AF during the baseline CEMRA only, one patient during follow up CE-MRA only, and one patient during both. All patients were asymptomatic with regard to PV stenosis. Seventeen patients had two right (upper and lower) and two left (upper and lower) PVs. Six patients lacked a left lower PV. There was a decrease in every measure of PV size after AF ablation, with the greatest reduction in CSA (table 1 ). No PV had a diameter reduction of $\geqslant 50 \%$ but seven $(8.1 \%)$ PVs had a reduction of $\geqslant 25 \%$. In comparison, nine $(10.5 \%)$ PVs had a CSA reduction of $\geqslant 50 \%$ and $16(18.6 \%)$ PVs had a reduction of $\geqslant 25 \%$ and $<50 \%$. There was no change in the left atrial dimension (40 (7) $\mathrm{mm}$ $v 39$ (6) $\mathrm{mm}, \mathrm{p}=0.25$ ).

We had data for 15 patients regarding the number of radiofrequency ablations and the duration of radiofrequency application for each PV. Standard correlation showed no linear relation between any PV size parameter and either the number of radiofrequency ablations or the duration of radiofrequency application $(r<0.2, \mathrm{p}>0.15$ for all). The mean number of ablations for each PV was 11 (10) seconds (median 8) and the mean duration of radiofrequency application for each PV was 262 (237) seconds (median 210). There were no significant differences in either the number of ablations or the ablation duration between the PVs ( $p>0.1$ for all). There were no significant differences in any PV size parameter for PVs that received $>8$ radiofrequency ablations $(n=25)$ compared with those that received $\leqslant 8$ radiofrequency ablations $(\mathrm{n}=30)$ or for PVs that received radiofrequency ablation for $>210$ seconds $(\mathrm{n}=27)$ compared with those that received radiofrequency ablation for $\leqslant 210$ seconds $(\mathrm{n}=28, \mathrm{p}>0.1$ for all $)$.

\section{DISCUSSION}

In the absence of clinical PV stenosis, we found a significant reduction in all measures of $\mathrm{PV}$ size after AF ablation with the most pronounced reduction in CSA. As AF ablation procedures become more common, it becomes increasingly important to understand its potential risks. Although clinical PV stenosis remains rare, ${ }^{2}$ reductions in PV diameter have recently been reported in up to a quarter of PVs after ablation. ${ }^{4}$ Our data suggest that these diameter measurements may significantly underestimate the prevalence of subclinical PV narrowing after AF ablation.

We found significant reductions in all measures of PV size 1.3 months after PV ablation compared with baseline values. The reduction in PV size was most pronounced by measures of CSA. Almost one third of PVs had $a \geqslant 25 \%$ reduction in CSA. Although the potential clinical significance of asymptomatic reductions in PV size is unknown, we found that the PV diameter may underestimate the frequency and severity of

Abbreviations: AF, atrial fibrillation; CE-MRA, contrast enhanced three dimensional magnetic resonance angiography; CSA, cross sectional area; $\mathrm{PV}$, pulmonary vein 
Table 1 Pulmonary vein measurements before and after ablation

\begin{tabular}{|c|c|c|c|c|c|}
\hline \multirow[b]{2}{*}{ Measurement } & \multicolumn{5}{|l|}{ Pulmonary vein } \\
\hline & All $(n=86)$ & Lower left $(n=17)$ & Right lower $(n=23$ ) & Left upper ( $n=223$ ) & Right upper ( $n=23$ ) \\
\hline \multicolumn{6}{|c|}{ Maximum diameter $(\mathrm{mm})$} \\
\hline Before & $20.8(5.6)$ & $18.1(2.2)$ & $20.0(5.3)$ & $20.3(6.6)$ & $24.3(5.0)$ \\
\hline After & $19.2(5.2)$ & $16.1(3.2)$ & $19.0(4.0)$ & $18.5(6.4)$ & $22.5(4.4)$ \\
\hline Difference* & 1.6 (1.1 to 2.2 ) & $2.0(0.5$ to 3.5$)$ & $1.0(-0.1$ to 2.1$)$ & $1.8(0.7$ to 2.9$)$ & $1.8(0.6$ to 3.1$)$ \\
\hline p Value & $<0.001$ & 0.011 & 0.071 & 0.002 & 0.006 \\
\hline \multicolumn{6}{|l|}{ Perimeter $(\mathrm{mm})$} \\
\hline Before & $57(14)$ & $47(6)$ & $57(14)$ & $55(15)$ & $66(12)$ \\
\hline After & $52(13)$ & $41(8)$ & $54(10)$ & $48(15)$ & $61(10)$ \\
\hline Difference* & $5.2(3.7$ to 6.6$)$ & $6.0(2.8$ to 9.2$)$ & $2.6(-0.5$ to 5.6$)$ & $7.3(5.0$ to 9.6$)$ & $5.1(1.9$ to 8.3$)$ \\
\hline $\mathrm{p}$ Value & $<0.001$ & 0.001 & 0.093 & $<0.001$ & 0.003 \\
\hline \multicolumn{6}{|c|}{ Cross sectional area $\left(\mathrm{mm}^{2}\right)$} \\
\hline Before & $236(118)$ & $154(48)$ & $236(99)$ & $213(107)$ & $320(131)$ \\
\hline After & $186(91)$ & $118(43)$ & $213(69)$ & $162(95)$ & $257(85)$ \\
\hline Difference* & 43 (29 to 58 ) & 36 (17 to 55$)$ & 23 (3 to 42 ) & 51 (35 to 67) & 62 (16 to 108$)$ \\
\hline $\mathrm{p}$ Value & $<0.001$ & $<0.001$ & 0.027 & $<0.001$ & 0.011 \\
\hline
\end{tabular}

subclinical PV stenosis compared with CSA. Prior studies that relied on diameter measurements ${ }^{4}$ may have underestimated the true prevalence of asymptomatic reduction in PV size after AF ablation.

We did not find a relation between reduction in PV size and two measures of the intensity of radiofrequency ablation. This differs from a prior report that found an association between the radial angle of energy delivery and the severity of the reduction in PV diameter after $\mathrm{AF}$ ablation. ${ }^{4}$ In addition to using different measures for the intensity of ablation, both the present study and the prior study assessed relatively few patients. Narrowing may progress in up to $10 \%$ of patients after one month. ${ }^{2}$ A larger cohort may be required to determine the true relation between the intensity of ablation and changes in PV size after AF ablation.

\section{ACKNOWLEDGEMENTS}

Dr Hauser is supported in part by the Clinical Investigator Training Program, Beth Israel Deaconess Medical Center-Harvard/MIT Health Sciences and Technology, in collaboration with Pfizer Inc.

\section{Authors' affiliations}

T H Hauser, S B Yeon, S McClennen, G Katsimaglis, K V Kissinger, M E Josephson, W J Manning*, The Department of Medicine, Cardiovascular Division, Beth Israel Deaconess Medical Center and Harvard Medical School, Boston, Massachusetts, USA
N M Rofsky, Department of Radiology, Beth Israel Deaconess Medical Center and Harvard Medical School

*Also at the Department of Radiology

Correspondence to: Dr Thomas H Hauser, Cardiovascular Division, Beth Israel Deaconess Medical Center, 330 Brookline Avenue, RW-453, Boston, MA 02215, USA;

thauser@bidmc.harvard.edu

Accepted 11 June 2004

\section{REFERENCES}

1 Haissaguerre $M$, Jais $P$, Shah DC, et al. Spontaneous initiation of atrial fibrillation by ectopic beats originating in the pulmonary veins. N Engl J Med 1998:339:659-66

2 Saad EB, Rossillo A, Saad CP, et al. Pulmonary vein stenosis after radiofrequency ablation of atrial fibrillation: functional characterization, evolution, and influence of the ablation strategy. Circulation 2003; 108:3102-7.

3 Hauser TH, Yeon SB, McClennen S, et al. A method for the determination of proximal pulmonary vein size using contrast enhanced magnetic resonance angiography. J Cardiovasc Magn Reson 2004;6:925-34.

4 Dill T, Neumann T, Ekinci $O$, et al. Pulmonary vein diameter reduction after radiofrequency catheter ablation for paroxysmal atrial fibrillation evaluated by contrast-enhanced three-dimensional magnetic resonance imaging. Circulation 2003;107:845-50.

5 Oral H, Knight BP, Ozaydin M, et al. Segmental ostial ablation to isolate the pulmonary veins during atrial fibrillation: feasibility and mechanistic insights. Circulation 2002; 106:1256-62. 